\title{
OPEN Modulation of circadian clock by crude drug extracts used in Japanese Kampo medicine
}

\author{
Manhui Zhang ${ }^{1,2}$, Kohei Kobayashi ${ }^{1,2}$, Haruki Atsumi ${ }^{1,2}$, Yuma Katada ${ }^{1,2}$, Yusuke Nakane ${ }^{1,2}$, \\ Junfeng Chen ${ }^{1,2}$, Ryo Nagano ${ }^{1,2}$, Naoya Kadofusa ${ }^{1}$, Taeko Nishiwaki-Ohkawa ${ }^{1,2}$, \\ Naohiro Kon ${ }^{1,2}$, Tsuyoshi Hirota ${ }^{1}$, Ayato Sato ${ }^{1}$, Toshiaki Makino ${ }^{3}$ \& Takashi Yoshimura ${ }^{1,2 \bowtie}$
}

Circadian rhythm is an approximately $24 \mathrm{~h}$ endogenous biological rhythm. Chronic disruption of the circadian clock leads to an increased risk of diabetes, cardiovascular disease, and cancer. Hence, it is important to develop circadian clock modulators. Natural organisms are a good source of several medicines currently in use. Crude drugs used in Japanese traditional Kampo medicine or folk medicines are an excellent source for drug discovery. Furthermore, identifying new functions for existing drugs, known as the drug repositioning approach, is a popular and powerful tool. In this study, we screened 137 crude drug extracts to act as circadian clock modulators in human U2OS cells stably expressing the clock reporter Bmal1-dLuc, and approximately $12 \%$ of these modulated the circadian rhythm. We further examined the effects of several crude drugs in Rat-1 fibroblasts stably expressing Per2luc, explant culture of lung from Per2::Luciferase knockin mice, and zebrafish larvae in vivo. Notably, more than half of the major ingredients of these crude drugs were reported to target AKT and its relevant signaling pathways. As expected, analysis of the major ingredients targeting AKT signaling confirmed the circadian clock-modulating effects. Furthermore, activator and inhibitor of AKT, and triple knockdown of $A K T$ isoforms by siRNA also modulated the circadian rhythm. This study, by employing the drug repositioning approach, shows that Kampo medicines are a useful source for the identification of underlying mechanisms of circadian clock modulators and could potentially be used in the treatment of circadian clock disruption.

The circadian rhythm is an approximately $24 \mathrm{~h}$ cell-autonomous biological rhythm observed in virtually all living organisms. The mammalian circadian clock involves a transcription-translation feedback loop in which CLOCK and BMAL1 heterodimers activate the PERIOD (PER) and CRYPTOCHROME (CRY) genes, which then provide feedback and repress their transcriptions $s^{1,2}$. It has been reported that chronic circadian disruption is associated with many diseases, such as cardiovascular and cerebrovascular diseases ${ }^{3}$, cancer $^{4}$, immune $^{5}$, and metabolic disorders ${ }^{6}$.

The development of new pharmaceuticals is time-consuming and expensive. Therefore, identifying new functions for existing drugs, known as the drug repositioning approach, is a popular and powerful approach ${ }^{7}$. Crude drugs used in traditional Japanese Kampo medicine are the major sources of new chemical entities for drug discovery ${ }^{8}$. In this study, we screened 137 crude drug extracts to identify circadian clock modulators using a human osteosarcoma U2OS cell line stably expressing the clock reporter Bmall- $d L u c$ and identified 17 hit crude drugs. We noticed that more than half of the major ingredients of these crude drugs targeted AKT and relevant signaling pathways. Therefore, we examined the effects of the major ingredients targeted AKT. We then examined the effects of AKT activator and inhibitor, as well as the triple knockdown of AKT1/2/3 using siRNA.

\section{Results}

Identification of crude drug extracts that modulate the circadian clock. To identify circadian clock modulators, 137 crude drug extracts were screened in Bmal1-dLuc U2OS for primary screening ${ }^{7}$. Since crude drug extract is a mixture of many active ingredients and their effects could be highly variable, all crude

${ }^{1}$ Institute of Transformative Bio-Molecules (WPI-ITbM), Nagoya University, Furo-cho, Chikusa-ku, Nagoya 464-8601, Japan. ${ }^{2}$ Laboratory of Animal Integrative Physiology, Graduate School of Bioagricultural Sciences, Nagoya University, Furo-cho, Chikusa-ku, Nagoya 464-8601, Japan. ${ }^{3}$ Department of Pharmacognosy, Graduate School of Pharmaceutical Sciences, Nagoya City University, 3-1 Tanabe-Dori, Mizuho-ku, Nagoya 467-8603, Japan. ${ }^{\circledR}$ email: takashiy@agr.nagoya-u.ac.jp 

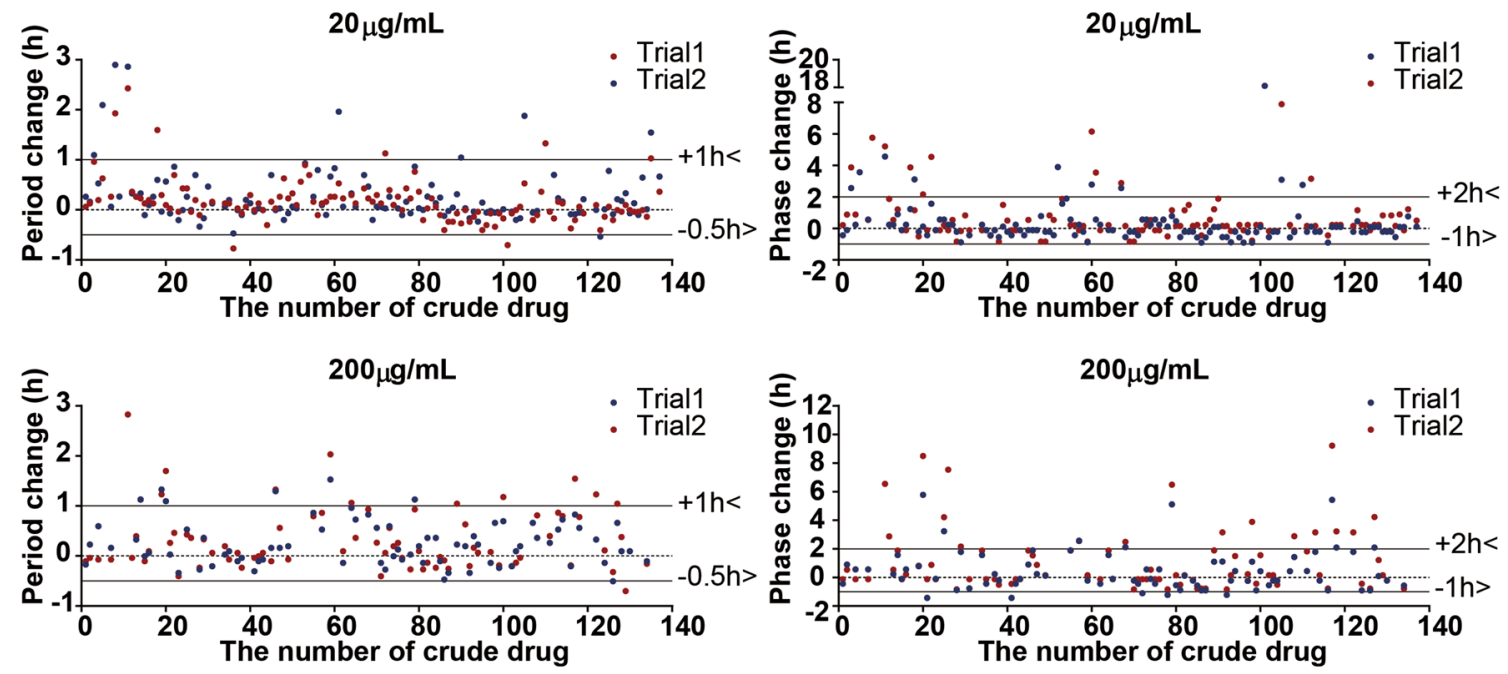

Figure 1. Screening of crude drug extracts for circadian clock modulators. A total of 137 crude drug extracts were screened in Bmal1-dluc U2OS cells at $20 \mu \mathrm{g} / \mathrm{mL}$ (top) and $0.20 \mathrm{mg} / \mathrm{mL}$ (bottom) for circadian period (left) and phase (right) changes. (Left) Two independent trials identified two crude drugs at $20 \mu \mathrm{g} / \mathrm{mL}$ and four crude drugs at $0.20 \mathrm{mg} / \mathrm{mL}$ that show consistent period lengthening effects by 1 or more hours. (Right) This screening also identified five crude drugs at $20 \mu \mathrm{g} / \mathrm{mL}$ and eight crude drugs at $0.20 \mathrm{mg} / \mathrm{mL}$ that show consistent phase changing effects by 2 or more hours.

\begin{tabular}{|c|c|c|c|c|}
\hline Number & Name of crude drugs & Origin & Lot number & Distributor \\
\hline 3 & Artemisiae Capillaris Flos & The dried capitulum of Artemisia capillaris Thunberg & $0 \mathrm{H} 03 \mathrm{M}$ & Daiko \\
\hline 11 & Polygalae Radix & The dried root bark of Polygala tenuifolia Willdenow & $3 \mathrm{I} 17 \mathrm{M}$ & Daiko \\
\hline 14 & Puerariae Radix & $\begin{array}{l}\text { The dried root of Pueraria montana var. lobata (Willd.) } \\
\text { Sanjappa \& Pradeep }\end{array}$ & C36361 & Tsumura \\
\hline 19 & Platycodi Radix & The dried root of Platycodon grandiflorum A. De Candolle & I420115 & Uchida \\
\hline 20 & Chrysanthemi Flos & The dried capitulum of Chrysanmum morifolium Ramatulle & $2 \mathrm{H} 28$ & Daiko \\
\hline 25 & Schizonepetae Spica & The dried spike of Nepeta tenuifolia Bentham & 7J03 & Daiko \\
\hline 46 & Paeoniae Radix & The dried root of Paeonia lactiflora Pallas & $5 \mathrm{D} 08 \mathrm{M}$ & Daiko \\
\hline 57 & Cicadae Periosrtacum & The dried larval exuvia of Cryptotympana atrata Stal & $5 \mathrm{C} 30$ & Daiko \\
\hline 60 & Perillae Herba & $\begin{array}{l}\text { The dried leaves and branches of Perilla frutescens Britton var. } \\
\text { crispa Decaisne }\end{array}$ & $8 \mathrm{~F} 16$ & Daiko \\
\hline 67 & Caryophylli Flos & $\begin{array}{l}\text { The dried flowering bud of Syzygium aromaticum Merrill et } \\
\text { Perry }\end{array}$ & $2 \mathrm{G} 31 \mathrm{M}$ & Daiko \\
\hline 68 & Uncariae Uncis Cum Ramulus & $\begin{array}{l}\text { The dried hook-bearing stem of Uncaria rhynchophylla } \\
\text { Miquel ex Haviland }\end{array}$ & $1010 \mathrm{C} 022701$ & Tochimoto \\
\hline 79 & Lonicerae Folium Cum Caulis & The dried leaves and stems of Lonicera japonica Thunberg & $5 \mathrm{I} 21$ & Daiko \\
\hline 105 & Alpiniae Officinari Rhizoma & The dried rhizome of Alpinia officinarum Hance & 023008001 & Tochimoto \\
\hline 118 & Perillae Fructus & $\begin{array}{l}\text { The dried fruit of Perilla frutescens Britton var. crispa } \\
\text { Decaisne }\end{array}$ & $8 \mathrm{~A} 09$ & Daiko \\
\hline 119 & Acorus Graminei Rhizoma & The dried rhizome of Acorus gramineus Aiton & P010701311 & Tochimoto \\
\hline 129 & Mume Fructus Praeparatus & The dried steamed fruit of Prunus mume Siebold et Zuccarini & 9D01 & Daiko \\
\hline 137 & Allii Chinense Bulbus & The bulbs of Allium chinense G.Don & $7 \mathrm{I} 18$ & Daiko \\
\hline
\end{tabular}

Table 1. List of hit crude drugs. All crude drugs are registered in the Japanese Pharmacopoeia 17th Edition or the Japanese standards for non-Pharmacopoeial crude drugs, 2015. Daiko Shoyaku (Nagoya, Japan), Tsumura (Tokyo, Japan), Uchida Wakanyaku (Tokyo, Japan), and Tochimoto Tenkaido (Osaka, Japan).

drug extracts were tested in triplicates at two different concentrations $(20 \mu \mathrm{g} / \mathrm{mL}$ and $0.20 \mathrm{mg} / \mathrm{mL})$ in a 384 -well plate format, and the experiments were repeated twice (Trials 1 and 2) to reduce the number of false positives. Two crude drug (Polygalae Radix and Allii Chinense Bulbus) extracts at $20 \mu \mathrm{g} / \mathrm{mL}$ and four crude drug (Pueraiae Radix, Platycodi Radix, Chrysanthemi Flos, and Paeoniae Radix) extracts at $0.20 \mathrm{mg} / \mathrm{mL}$ were identified to show period lengthening effects by one or more hours, respectively (Fig. 1, Table 1). Five crude drug (Artemisiae Capillaris Flos, Polygalae Radix, Perillae Herba, Caryophylli Flos, and Alpiniae Officinari Rhizoma) extracts at $20 \mu \mathrm{g} / \mathrm{mL}$ and eight crude drug (Chrysanthemi Flos, Schizonepetae Spica, Cicadae Periosrtacum, Uncariae Uncis Cum Ramulus, Lonicerae Folium Cum Caulis, Perillae Fruitus, Acorus Graminei Rhizoma, and Mume 
Fructus Praeparatus) extracts at $0.20 \mathrm{mg} / \mathrm{mL}$ were identified to show phase changing effects by two or more hours, respectively (Fig. 1, Table 1). To validate the 17 potential hit crude drug extracts, we performed secondary screening for dose-dependency and confirmed the period and/or phase-changing effects for all 17 crude drug extracts (Fig. 2). To determine whether the effects of crude drugs on the circadian clock were reporter gene-, cell type-, or tissue-specific, the effects of three representative hit crude drugs (Artemisaie Capillaris Flos, Perillae Herba, and Allii Chinense Bulbus) were examined in Rat-1 Per2-luc fibroblasts ${ }^{9}$ (Fig. 3A). The period lengthening and phase changing effects of the extracts of Artemisiae Capillaris Flos and Perillae Herba were consistent with the results obtained for U2OS cells. Interestingly, however, Allii Chinense Bulbus extract showed periodshortening effects. When we examined the effects of these three crude drug extracts in lung explant cultures from Per2::Luciferase knockin mice ${ }^{7}$, all three crude drug extracts showed period-lengthening effects (Fig. 3B). We also examined the effect of Allii Chinense Bulbus extract on the locomotor activity rhythm of zebrafish larvae in vivo under constant darkness and observed a period shortening effect and a tendency to phase change (Fig. 4). As we are aware that some of the compounds affect the bioluminescence amplitude through the activation or inhibition of luciferase activity but not through the circadian clock, we did not focus on the circadian amplitude in the present study.

Ingredients of hit crude drugs modulate the circadian rhythm. As previously mentioned, the crude drug extract is a mixture containing many active ingredients. In addition, because the administration of crude lysates sometimes affects the experimental results owing to the formation of macromolecular complexes and/or medium $\mathrm{pH}$ change $\mathrm{e}^{10}$, validation using known active ingredients is important. The well-known active ingredients for hit crude drugs are summarized in Table S1. To further understand the possible mode of action of these crude drugs, we searched for known targets of these active ingredients from previous literature and found that $\mathrm{AKT}, \mathrm{NFKB}$, and mTOR are the most common targets for these active ingredients (Table $\mathrm{S} 1$ ). To test whether the major ingredients that target $\mathrm{AKT}, \mathrm{NF}-\mathrm{\kappa B}$, and mTOR can modulate the circadian rhythm, the effects of eight major ingredients were examined in U2OS cells, and it was observed that treatment with these ingredients leads to significant changes in the circadian period and/or phase (Fig. 5).

Involvement of AKT signaling in the circadian clock. It is well established that the signaling pathways for NF- $\kappa \mathrm{B}, \mathrm{mTOR}$, and AKT are closely related, and AKT is a major signaling hub (Fig. 6A). Involvement of $A k t$ in the circadian clock has been reported in Drosophila ${ }^{11}$. There are three isoforms of AKT in mammals. However, analysis of the circadian behavior in Akt1-/- mice revealed a normal behavioral rhythm ${ }^{12}$. We examined the effects of AKT activator and inhibitor to explore the role of AKTs in the mammalian circadian clock. The AKT activator SC79 shortened the circadian period and advanced the phase at the highest dose (Fig. 6B). In contrast, the AKT inhibitor A-443654 shortened the circadian period at lower doses and lengthened the period at the highest dose (Fig. 6C). A-443654 also delayed the circadian phase in a dose-dependent manner (Fig. 6C). Furthermore, an siRNA-mediated triple knockdown of $A K T 1 / 2 / 3$ shortened the circadian period (Fig. 6D).

\section{Discussion}

Chronic disruption of the circadian clock due to shift work or travel across time zones has long-term consequences on human health, resulting in an increased risk of many diseases such as diabetes, cardiovascular disease, and cancer ${ }^{3-6}$. The development of new pharmaceuticals is costly and time-consuming, with a high rate of failure. Identifying new functions for existing drugs, known as the drug repositioning approach, is a popular and powerful approach, and crude drugs used in traditional Japanese Kampo medicine are major sources of new chemical entities for drug discovery ${ }^{7,8}$. Indeed, Motohashi et al. succeeded in identifying circadian clock modulators after screening 40 crude drugs ${ }^{13}$.

In this study, we screened 137 crude drug extracts, of which approximately $12 \%$ modulated circadian rhythms (Figs. 1 and 2, Table 1). In our previous study, using the US Food and Drug Administration (FDA)-approved drug library and the International Drug Collection (IDC) library, we found that approximately $5 \%$ of the existing drugs alter circadian rhythms ${ }^{7}$. The hit rate of Kampo medicines was much higher than that of other existing drug libraries. The reason for the high hit rate is probably because the crude drug extract is a mixture containing many active ingredients. Indeed, the effects of the major ingredients were not as strong as those of the original crude drugs (Fig. 5).

To further test whether these effects are specific to the reporter gene (Bmall-dLuc) or cell type (U2OS cells), we examined the effects of three representative hit crude drugs (Artemisiae Capillaris Flos, Perillae Herba, and Allii Chinense Bulbus) in Rat-1 fibroblasts expressing Per2-luc and lung explants from Per2::Luciferase knockin mice (Fig. 3). Although the effects of the extracts of Artemisiae Capillaris Flos and Perillae Herba were consistent with those observed in U2OS cells, Allii Chinense Bulbus extract showed the opposite period-shortening effects in Rat-1 cells (Fig. 3). When we examined the effects of Allii Chinense Bulbus extract on the locomotor rhythm of zebrafish larvae in vivo, we observed period shortening effects, as in the case of Rat-1 cells (Fig. 4). Discrepancies in the effects of crude drugs (i.e., period shortening or lengthening) between different reporters and cell types could be a result of the cell type-specific function of the circadian clock gene ${ }^{14}$ and/or the dissociation of clock gene rhythmicity within the cells/tissues ${ }^{15}$. However, as crude drug extracts are a mixture containing many active ingredients, it is also possible that the expression level of the drug targets could be different between different cells and tissues. Notably, tissue-dependent effects of crude drug extracts have also been observed ${ }^{13}$. This study clearly demonstrated the circadian clock modulating effects of crude drugs at the cellular, tissue, and organism levels.

When we searched for the potential targets of the identified ingredients in previous literature, we noticed that most of the ingredients targeted the NF- $\kappa \mathrm{B}, \mathrm{mTOR}$, or AKT signaling pathways (Table S1). Among them, $\mathrm{AKT}$ is known to be a major signaling hub (Fig. 6A). It is noteworthy that Akt signaling has been shown to affect 

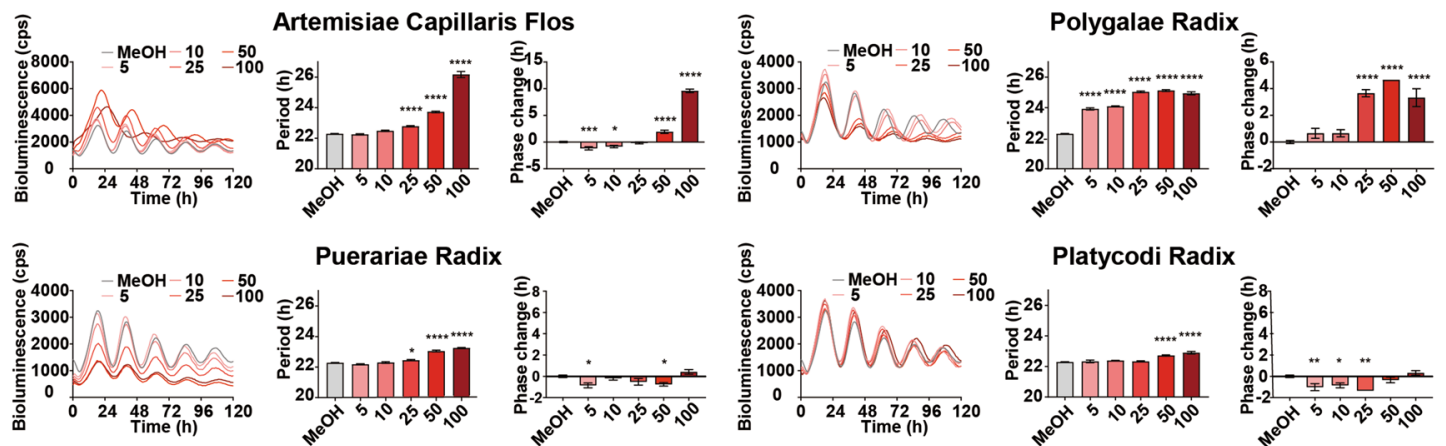

Puerariae Radix
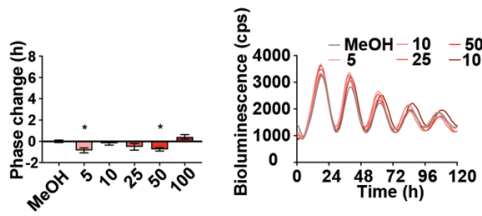

Platycodi Radix
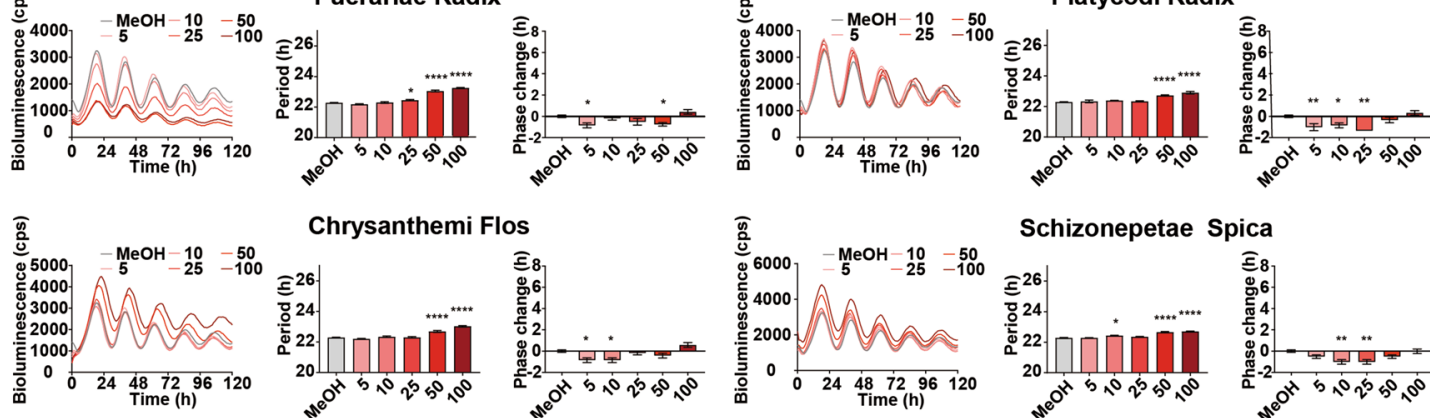

Chrysanthemi Flos
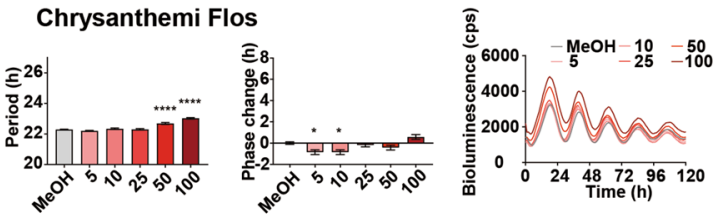

Schizonepetae Spica

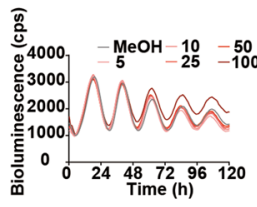

Paeoniae Radix
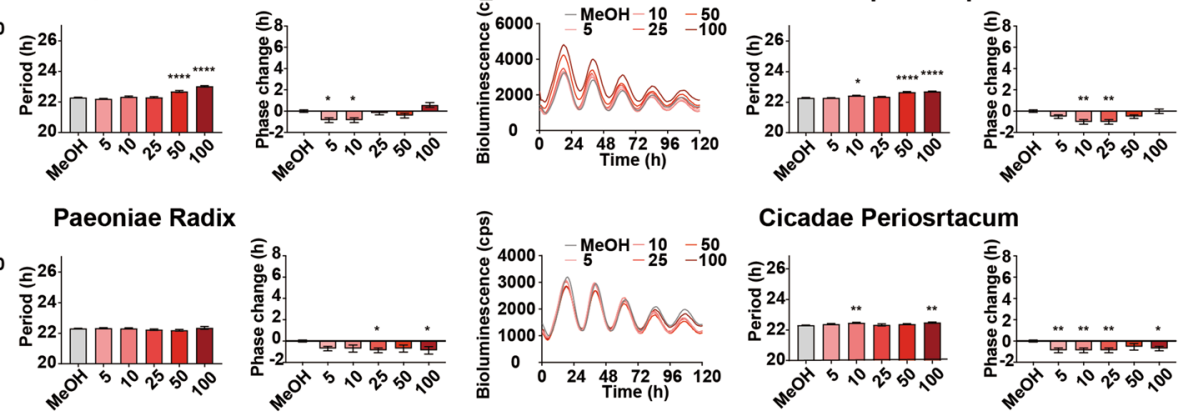

Cicadae Periosrtacum

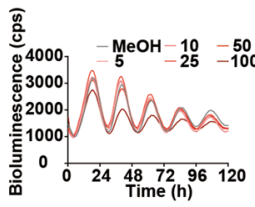

Perillae Herba
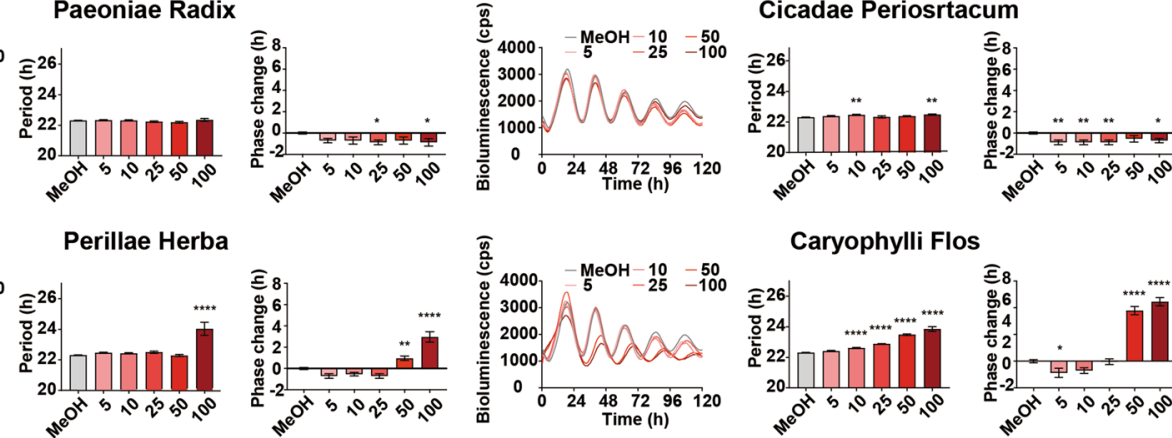

Caryophylli Flos

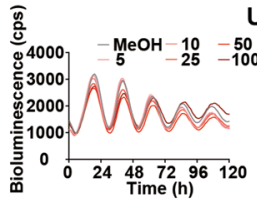

Uncariae Uncis Cum Ramulus

然
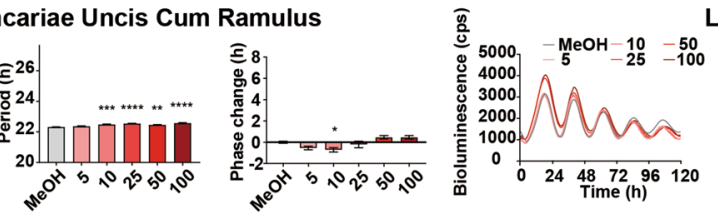

Lonicerae Folium Cum Caulis
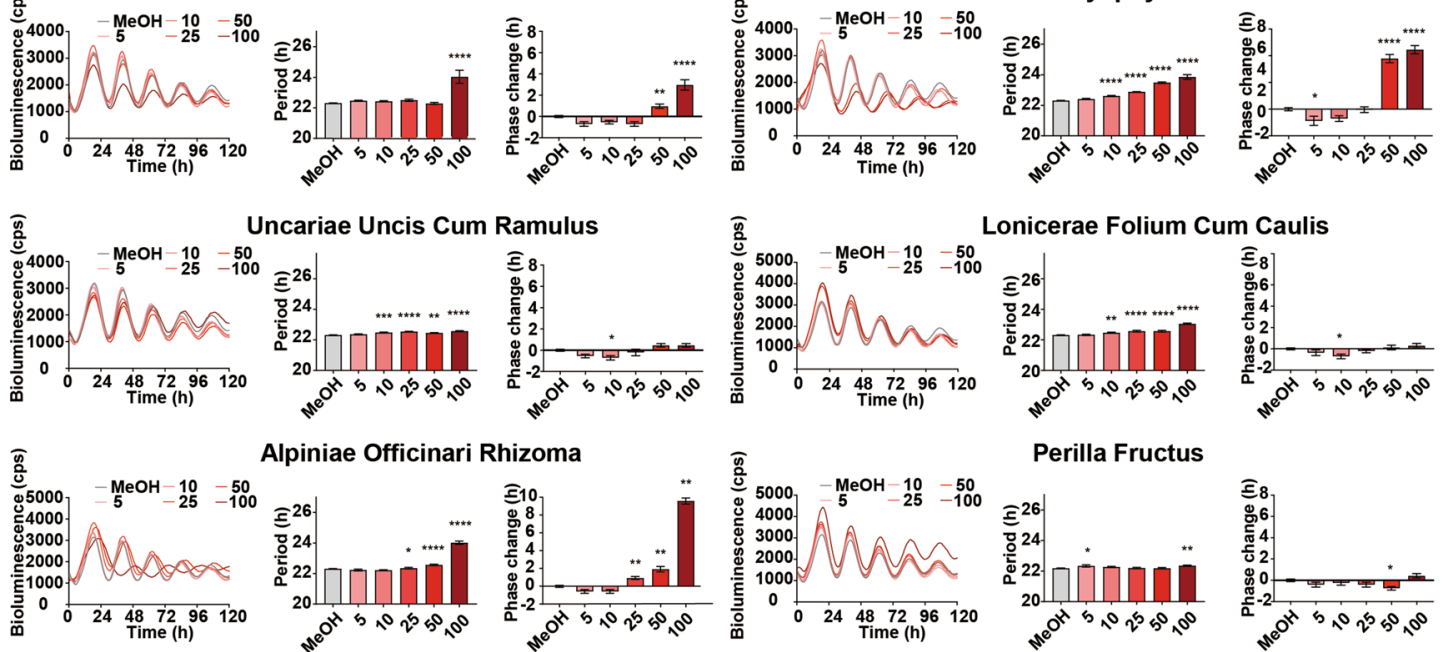

Alpiniae Officinari Rhizoma
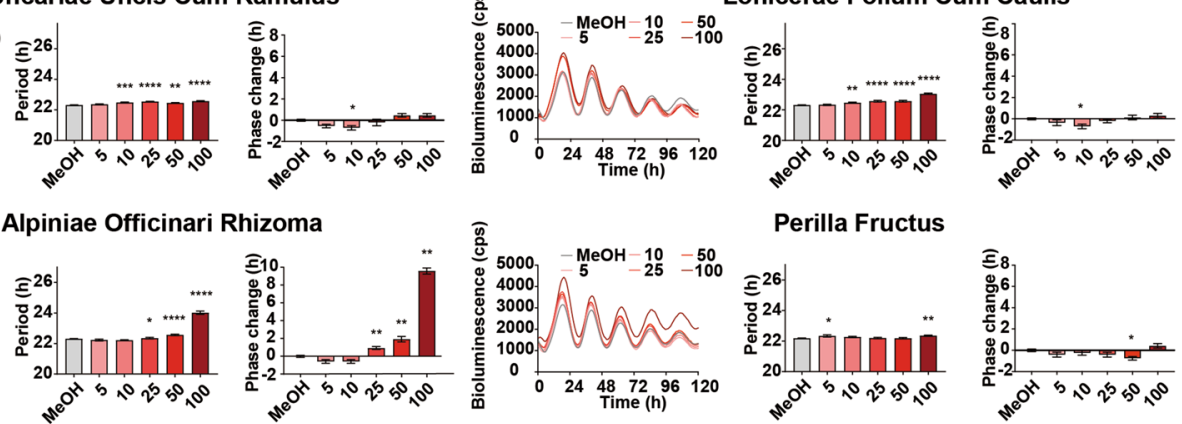

Perilla Fructus
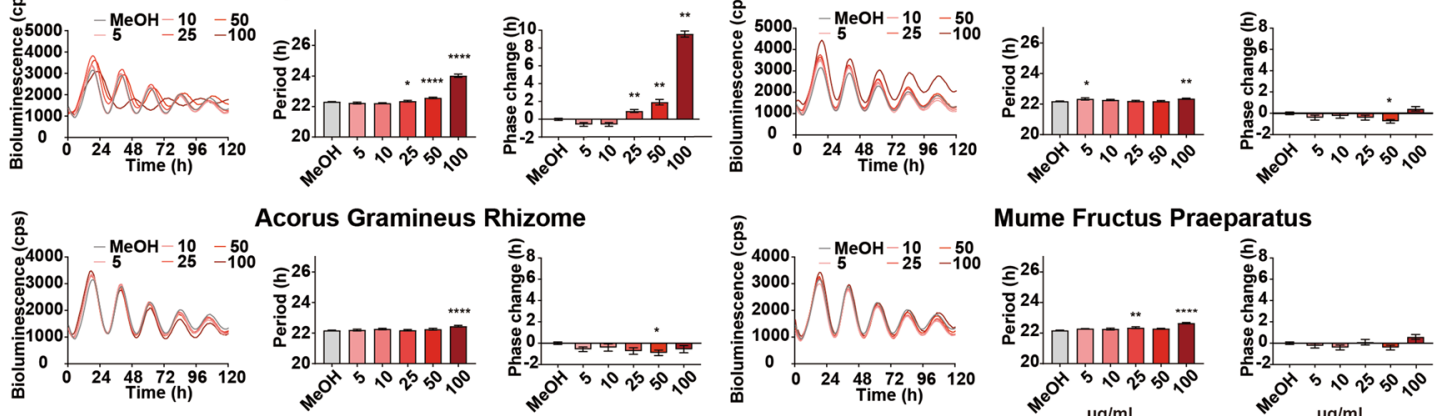

Acorus Gramineus Rhizome
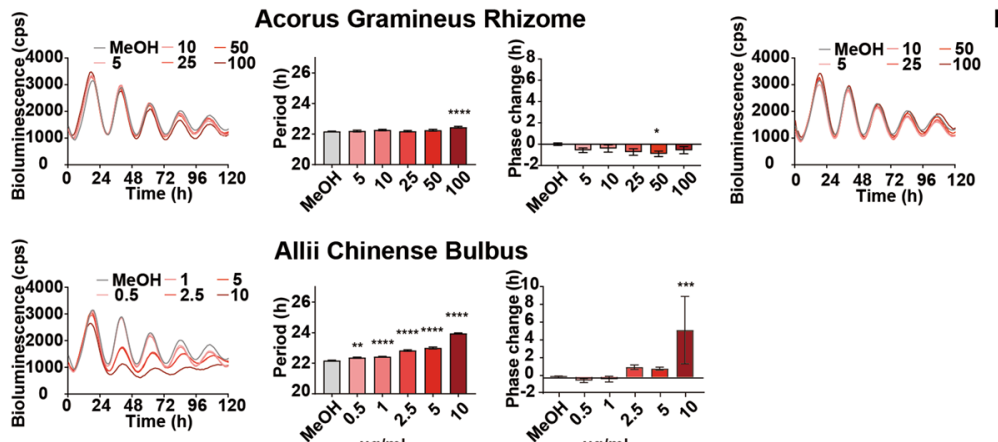

Mume Fructus Praeparatus
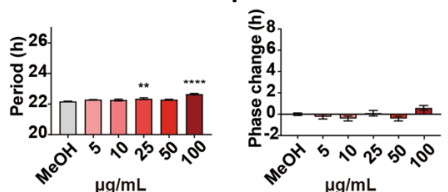

Allii Chinense Bulbus
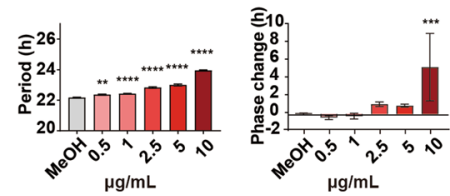

Figure 2. Dose-dependent effect of 17 hit crude drug extracts in U2OS cells expressing Bmal1-dLuc. Luminescent traces (left) and dose-dependent effects on the circadian period (middle) and phase (right) in $\mathrm{U} 2 \mathrm{OS}$ cells. Values are averages of six replicates \pm SD and were analyzed using one way ANOVA, followed by Dunnett's multiple comparisons test $\left({ }^{*} p<0.05,{ }^{* *} p<0.01,{ }^{* * *} p<0.001,{ }^{* * * *} p<0.0001\right)$. 
A

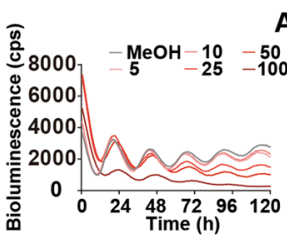

Artemisiae Capillaris Flos
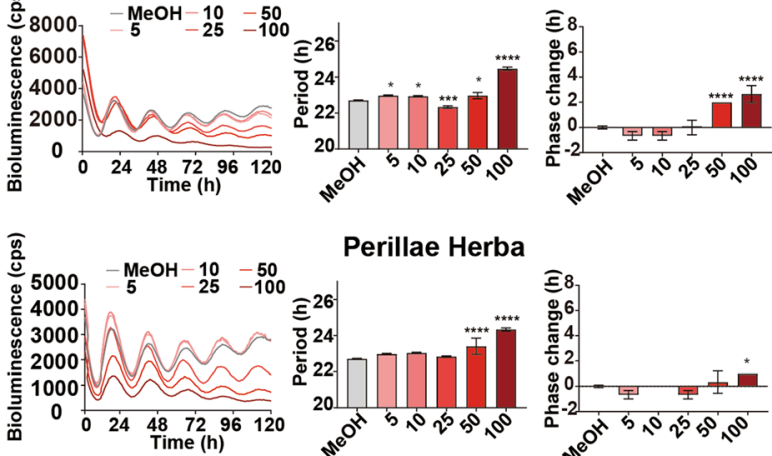

Perillae Herba
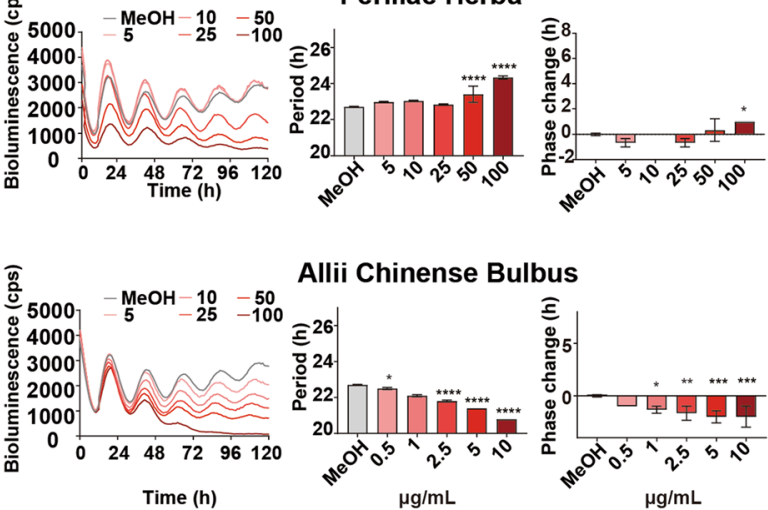

$\mathrm{B}$
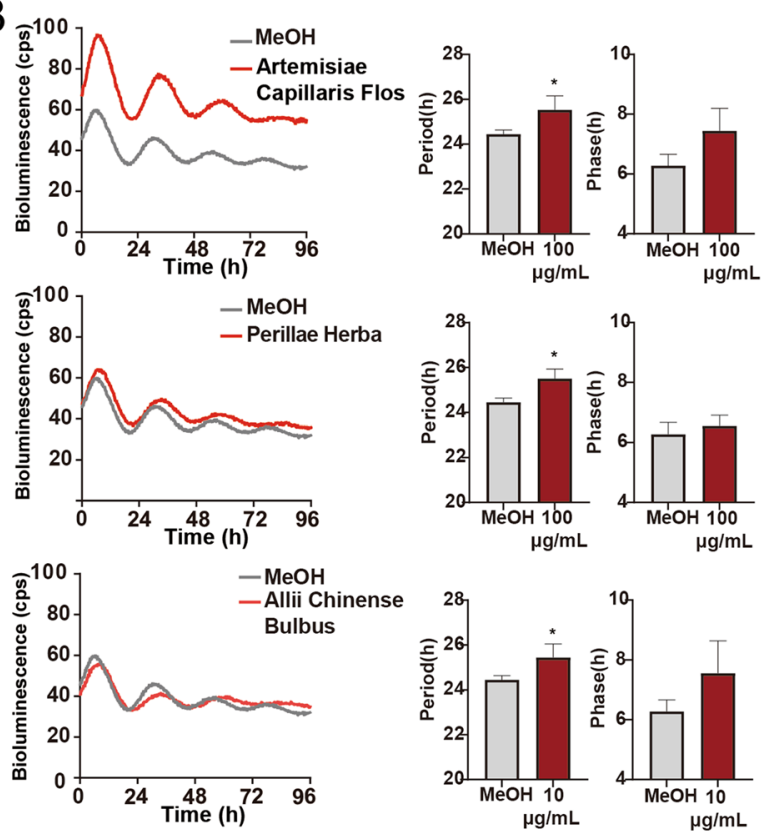

Figure 3. Effect of three crude drug extracts on Rat-1 fibroblasts expressing Per2-luc and lung explants from Per2::Luciferase knockin mice. (A) Luminescent traces (left) and dose-dependent effects on the circadian period (middle) and phase (right) in Rat-1 cells. Values are averages of three replicates \pm SD and were analyzed using one-way ANOVA, followed by Dunnett's multiple comparisons test $\left({ }^{*} p<0.05,{ }^{* *} p<0.01,{ }^{* * *} p<0.001,{ }^{* * *}\right.$ $p<0.0001$ ). (B) Effects of the extracts of Artemisiae Capillaris Flos (top), Perillae Herba (middle), and Allii Chinense Bulbus (bottom) on lung explants of Per2::Luciferase knockin mice. Luminescent traces (left) and effects on period (middle) and phase (right). Values are averages of three replicates \pm SD and were analyzed using Student's t-test. $\left({ }^{*} p<0.05\right)$.

\section{A}

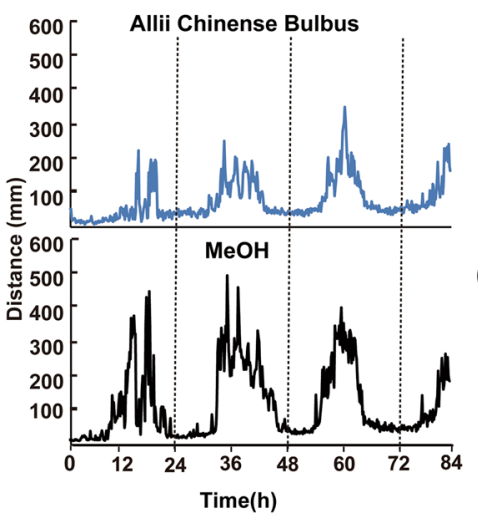

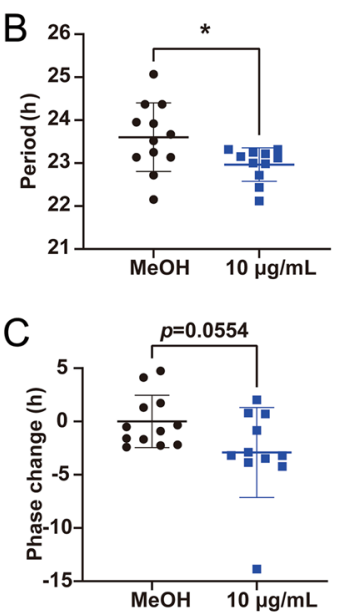

Figure 4. Effects of Allii Chinense Bulbus on locomotor activity rhythms of zebrafish larvae. (A) Locomotor activity rhythms under Allii Chinense Bulbus extract $(10 \mu \mathrm{g} / \mathrm{mL})$ and $\mathrm{MeOH}$ control. The moving distance $(\mathrm{mm})$ per $10 \mathrm{~min}$ were averaged and plotted $(\mathrm{n}=11,12)$. $(\mathbf{B}, \mathbf{C})$ Effects on circadian period $(\mathbf{B})$ and phase $(\mathbf{C})$ are shown. Values are average $\pm \mathrm{SD}$ and were analyzed by Student's $\mathrm{t}$-test $\left(\mathrm{n}=11,12,{ }^{\star} p<0.05\right)$.

the circadian period in Drosophila ${ }^{11}$. However, there are three isoforms of AKT in mammals, and Akt1-/- mice show normal behavioral rhythms ${ }^{12}$. In the present study, we found period and phase changes induced by AKT activator and inhibitor. We also confirmed a significant change in the circadian period in the triple knockdown of $A K T$ isoforms, suggesting the involvement of AKT signaling in the mammalian circadian clock (Fig. 6). Notably, PI3K, one of the signaling molecules upstream of the AKT signaling pathway, regulates feeding-mediated entrainment of the peripheral clock ${ }^{16}$. Furthermore, the CLOCK protein has recently been reported to be the substrate of $\mathrm{AKT}^{17}$. Further analysis of the precise mode of action of Kampo medicines may uncover the role of the AKT signaling pathway in the circadian clock. 

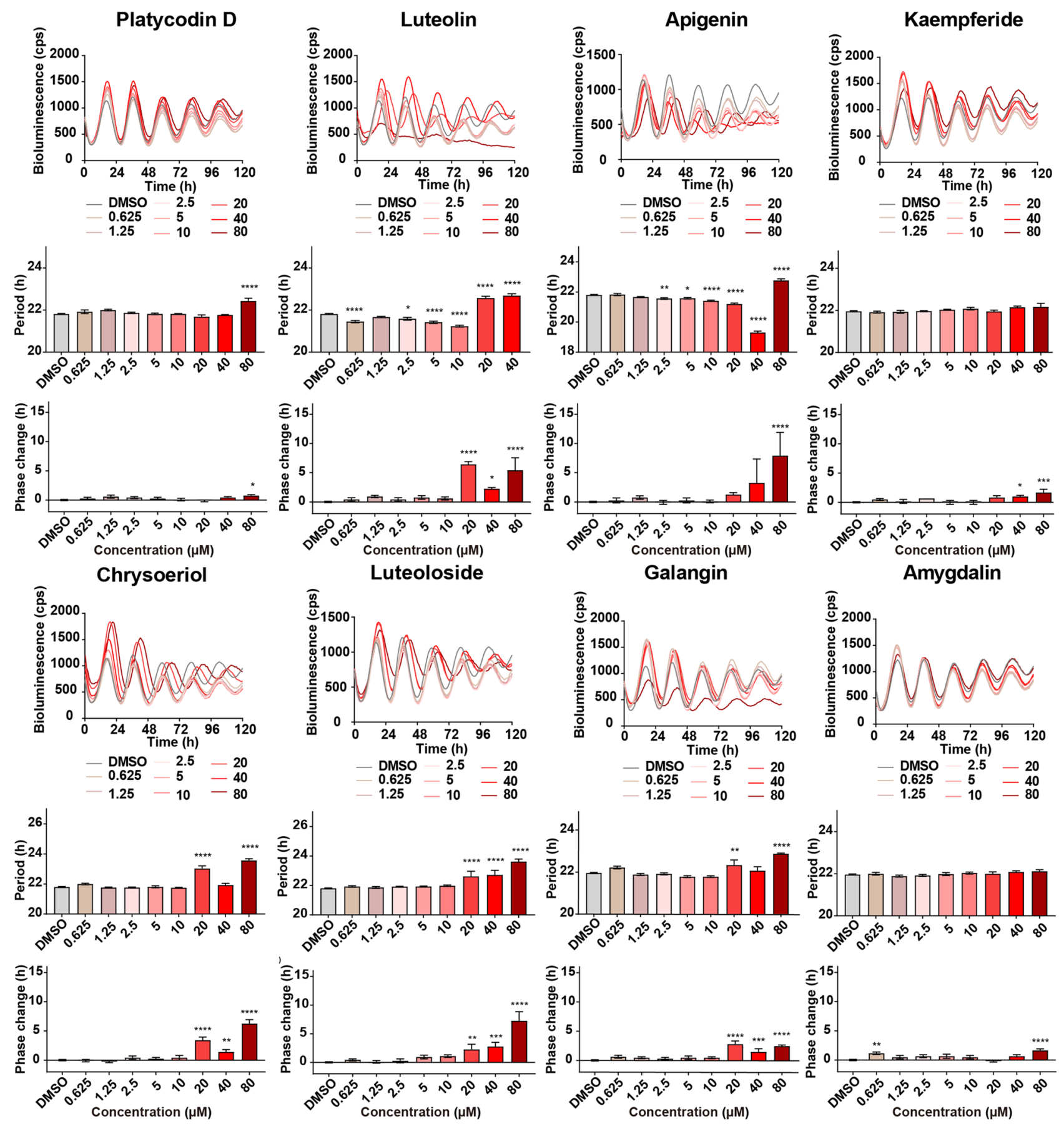

Figure 5. Effects of 8 major ingredients of hit crude drugs. Luminescent traces (top) and dose-dependent effects on the circadian period (middle) and phase (bottom) in U2OS cells. Values are the mean of six replicates \pm SD and were analyzed using one way ANOVA, followed by Dunnett's multiple comparisons test $\left({ }^{\star} p<0.05,{ }^{* *} p<0.01,{ }^{* *} p<0.001,{ }^{* * * *} p<0.0001\right)$.

In conclusion, the drug repositioning approach is a useful approach for understanding the underlying mechanisms of the circadian clock. Furthermore, tenuifolin, one of the main active ingredients in Polygalae Radix, has been reported to enhance sleep in mice $^{18}$. Thus, a drug repositioning approach using Kampo medicines is a useful approach for identifying potential therapeutic treatments for circadian misalignment.

\section{Materials and methods}

Crude drugs. We selected 137 crude drugs that are frequently used as ingredients in traditional Japanese Kampo formulations, and purchased crude drugs that met the grade standards of the Japanese Pharmacopoeia 17th Edition or of non-pharmacopoeial crude drugs from Daiko Shoyaku (Nagoya, Japan), Tsumura (Tokyo, Japan), Uchida Wakanyaku (Tokyo, Japan), and Tochimoto Tenkaido (Osaka, Japan). The preparation of metha- 
A
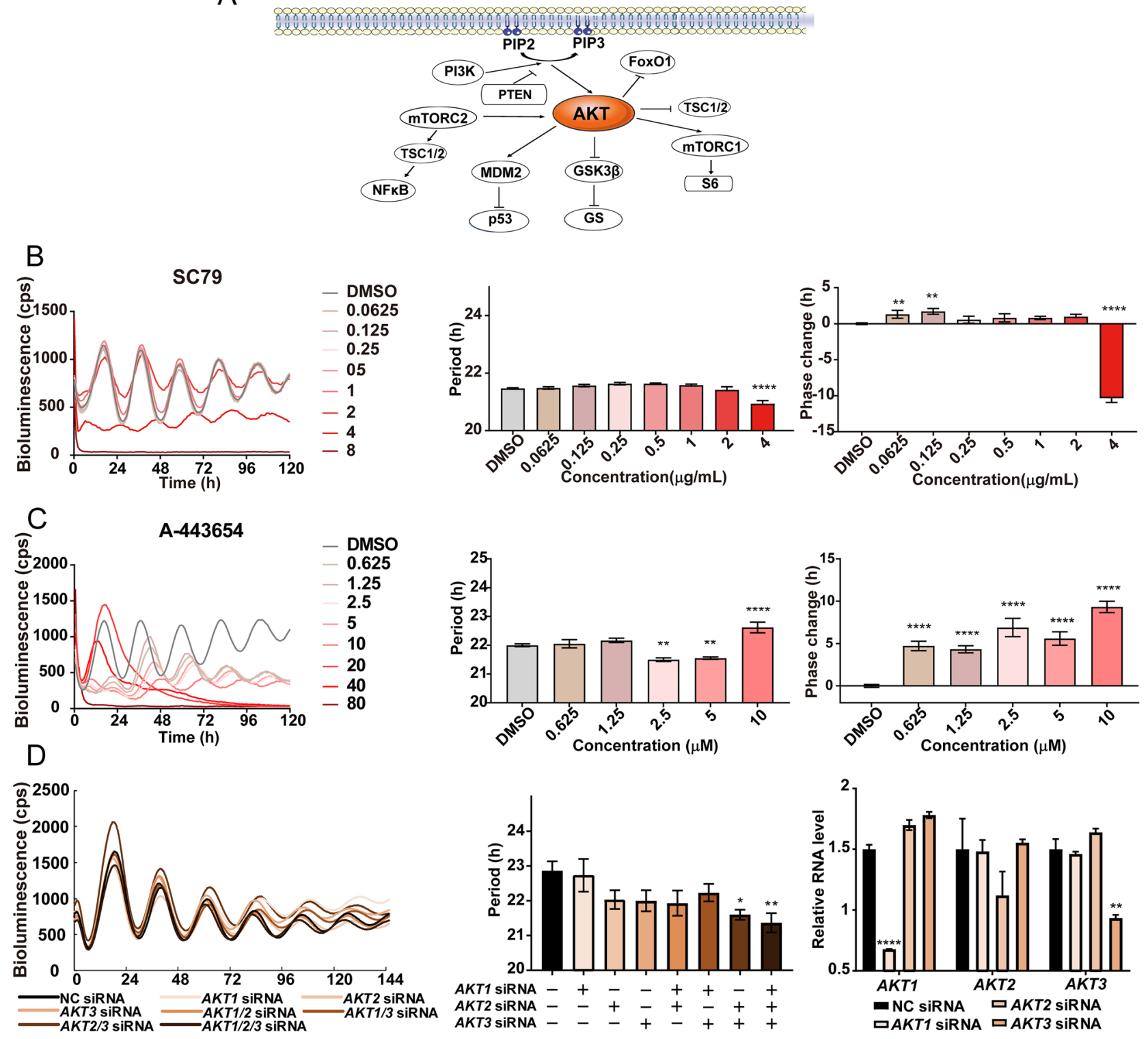

Figure 6. Involvement of AKT signaling in the circadian rhythm. (A) Schematic of interactions of the AKT pathway. (B,C) Effects of AKT activator SC79 (B) and AKT inhibitor A-443654 (C) at different concentration. Luminescent traces (left) and dose-dependent effects on period (middle) and phase (right) in U2OS cells. (D) Effects of the knockdown of $A K T 1 / 2 / 3$ by siRNA. U2OS cells were transfected with negative control (NC) siRNA, AKT1 siRNA, AKT2 siRNA, or AKT3 siRNA. Luminescent traces (left) and effects on circadian period (middle) in U2OS cells. Efficiency of knockdown of AKT was confirmed using RT-qPCR (right). Values are the averages of six replicates \pm SD and were analyzed using one way ANOVA, followed by Dunnett's multiple comparisons test $\left({ }^{*} p<0.05,{ }^{* *} p<0.01,{ }^{* * *} p<0.001,{ }^{* * *} p<0.0001\right)$.

nol extracts was described in our previous report ${ }^{8}$. Each extract was suspended in methanol at a concentration of $100 \mathrm{mg} / \mathrm{mL}$, and stored at $-20^{\circ} \mathrm{C}$. The origins and the distributors of the hit crude drugs are listed in Table 1.

Chemicals. Platycodin D (cat no. CFN98134), luteolin (cat no. CFN98784), luteoloside (cat no. CFN98565), galangin (cat no. CFN98918), kaempferide (cat no. CFN98782), and chrysoeriol (cat no. CFN98785) were purchased from ChemFaces (Wuhan, China). Amygdalin (cat no. A0443-1G) was purchased from Tokyo Chemical Industry (Tokyo, Japan). Apigenin (cat no. 010-18914) was obtained from Fujifilm Wako Pure Chemicals (Osaka, Japan). The AKT inhibitor, A-443654 (cat no. HY-10425), and the activator, SC79 (cat no. HY-18749) were purchased from MedChemExpress (Monmouth Junction, NJ, USA). Each chemical was suspended in DMSO at a concentration of $5 \mathrm{mM}$, stored at $-20^{\circ} \mathrm{C}$.

Cell culture. Human U2OS cell (HTB-96) and Rat-1 cell were obtained from ATCC (American Type Culture Collection). Human U2OS cells containing a Bmal1- $d L u c$ reporter gene and Rat-1 cells containing Per2-Luc 
reporter gene were cultured in Dulbecco's minimum essential medium (DMEM; D2902, Sigma-Aldrich, St. Louis, MI, USA) supplemented with $10 \%$ fetal calf serum (FBS,

FB-1290/500; Biosera, Rue Lacaille, Nuaillen, France), 2 mM L-glutamine, $100 \mathrm{U} / \mathrm{mL}$ penicillin, and $100 \mu \mathrm{g} /$ mL streptomycin (Pen Strep, 15070063, Thermo Fisher Scientific, Waltham, MA, USA) as previously described ${ }^{7}$.

Chemical screening. U2OS cells were seeded at a density of 4,000 cells/well in 384-well plates and incubated for 2 days until confluent. Using a Caliper Life Sciences ALH 3000 Workstation, crude drug extracts were diluted to $20 \mu \mathrm{g} / \mathrm{mL}$ and $0.20 \mathrm{mg} / \mathrm{mL}$ respectively, in assay media for measuring luminescence. This resulted in a final $\mathrm{MeOH}$ concentration of $0.2 \%$ in the control and drug-treated samples. Multiple doses $(0.63,1.3,2.5,5.0$, $10,20,40$, and $80 \mu \mathrm{M})$ of ingredients and AKT inhibitor A-443654 were analyzed, while AKT activator SC79 was analyzed at eight different doses $(0.063,0.13,0.25,0.5,1.0,2.0,4.0,8.0 \mu \mathrm{g} / \mathrm{mL})$. Luminescent assay media (also called "air" or recording media) was composed of DMEM, $10 \mathrm{mM}$ HEPES, $3.5 \mathrm{~g} / \mathrm{L}$ D-glucose, $0.35 \mathrm{~g} / \mathrm{L}$ sodium bicarbonate, $100 \mathrm{U} / \mathrm{mL}$ penicillin, $100 \mu \mathrm{g} / \mathrm{mL}$ streptomycin, 2\% B27 (Life Technologies, Carlsbad, CA, USA), $0.1 \mathrm{mM}$ luciferin (Fujifilm), and $100 \mathrm{nM}$ dexamethasone (Sigma). Diluted extracts were pipetted into triplicate wells of a 384-well plate containing U2OS cells (prepared above), and bioluminescence was monitored for 1 week using a Churitsu CL384 Series luminometer (Churitsu Electric Corporation, Nagoya, Japan). The circadian period and phase were determined using NINJA SL00-01 software for time series analysis (Churitsu Electric Corporation), and potential hit compounds were identified based on changes in the circadian period and/or phase.

Lung explant culture. Lungs were dissected from male Per2::Luciferase knockin mice and cultured in $35 \mathrm{~mm}$ dishes containing Millicell cell culture inserts (Millipore) in assay media to measure luminescence, as previously described ${ }^{7}$. The dishes were sealed with silicon grease and parafilm and bioluminescence was measured using a LumiCycle 32 (Actimetrics). The circadian period and phase were determined using LumiCycle Analysis software (Actimetrics). Note that phase-delays and -advances are indicated by positive and negative values, respectively.

Zebrafish behavior assay. Zebrafish (Danio rerio) larvae ( $3.5 \mathrm{dpf})$ were placed in each well of a $96 \mathrm{deep}-$ well plate (96 Well Clear Assay Plate Pyramid Flat Bottom); $800 \mu \mathrm{L}$ E3 medium $(5 \mathrm{mM} \mathrm{NaCl}, 0.17 \mathrm{mM} \mathrm{KCl}$, $0.33 \mathrm{mM} \mathrm{CaCl} \cdot 2 \mathrm{H}_{2} \mathrm{O}, 0.33 \mathrm{mM} \mathrm{MgSO} \cdot 7 \mathrm{H}_{2} \mathrm{O}, 10 \mathrm{mM}$ HEPES, pH 7.2) containing the compound $(10 \mu \mathrm{g} / \mathrm{mL})$ was added to each well. The plate was set on DanioVision (Noldus) and the behavior of the fish was tracked under constant darkness with a CCD camera with infrared light. The amount of activity (i.e., distance moved) per minute was calculated using the image processing function of EthoVision XT11 (Noldus). The free-running period and phase were calculated using Actogram J and MultiCycle (Actimetrics), respectively ${ }^{19}$. All animal studies were carried out in accordance with ARRIVE guidelines and all methods were in compliant with relevant guidelines and regulations and were approved by the Animal Experiment Committee of Nagoya University.

siRNA mediated knockdown. For gene knockdown analysis, small interfering RNA (siRNA) targeting the $A K T 1, A K T 2$, and $A K T 3$ sequences and non-targeting siRNA were obtained from Qiagen (FlexiTube GeneSolution siRNA, Hilden, Germany). These siRNAs were introduced singly, or as a mixture of two or three siRNAs into U2OS cells using a reverse transfection protocol in $35 \mathrm{~mm}$ dishes. Bioluminescence was monitored in a LumiCycle 32 (Actimetrix, Wilmette, IL, USA) for 1 week. The knockdown effect was verified using quantitative PCR. Reverse transcription was performed on total RNA (200 ng) using ReverTra Ace (Toyobo, Osaka, Japan) and oligo-dT primers. Samples contained SYBR Premix Ex Taq II (Takara, Kusatsu, Japan), $0.4 \mu$ M gene-specific primers (Table S2) and $2 \mu \mathrm{L}$ synthesized cDNA in $20 \mu \mathrm{L}$. qPCR was performed on an Applied Biosystems QuantStudio 3 Real-Time PCR System (Foster City, CA, USA) as follows: $95^{\circ} \mathrm{C}$ for $30 \mathrm{~s}$, followed by 40 cycles of $95^{\circ} \mathrm{C}$ for $5 \mathrm{~s}$ and $60^{\circ} \mathrm{C}$ for $30 \mathrm{~s} . \triangle \mathrm{Ct}$ was determined using $G A P D H$ as a housekeeping gene, and relative expression was calculated using the $\Delta \Delta \mathrm{Ct}$ method by comparing gene-specific siRNA samples to the negative siRNA control.

Statistical analysis. Statistical analysis was conducted using GraphPad Prism 9. Significance of differences was analyzed using one way analysis of variance (ANOVA) with Dunnett's multiple comparisons test or Student's t-test. Values are expressed as mean \pm SD.

Received: 14 June 2021; Accepted: 11 October 2021

Published online: 26 October 2021

\section{References}

1. Koike, N. et al. Transcriptional architecture and chromatin landscape of the core circadian clock in mammals. Science 338, 349-354 (2012).

2. Takahashi, J. S. Molecular components of the circadian clock in mammals. Diabetes Obes. Metab. 17(Suppl 1), 6-11 (2015).

3. Alibhai, F. J. et al. Disrupting the key circadian regulator CLOCK leads to age-dependent cardiovascular disease. J. Mol. Cell Cardiol. 105, 24-37 (2017).

4. Sulli, G. et al. Pharmacological activation of REV-ERBs is lethal in cancer and oncogene-induced senescence. Nature 553, 351-355 (2018).

5. Zhuang, X. D., Rambhatla, S. B., Lai, A. G. \& McKeating, J. Interplay between circadian clock and viral infection. J. Mol. Med. 95, 1283-1289 (2017). 
6. Hatori, M. et al. Time-restricted feeding without reducing caloric intake prevents metabolic diseases in mice fed a high-fat diet. Cell Metab. 15, 848-860 (2012).

7. Tamai, T. K. et al. Identification of circadian clock modulators from existing drugs. EMBO Mol. Med. 10, e8724 (2018).

8. Tashiro, Y. et al. Effects of osthol isolated from cnidium monnieri fruit on urate transporter 1. Molecules 23, 2837 (2018).

9. Kon, N. et al. Activation of TGF- $\beta$ /activin signalling resets the circadian clock through rapid induction of Dec1 transcripts. Nat. Cell Biol. 10, 1453-1469 (2008).

10. Pei, M., Xuan, C., Duan, X. \& Pei, X. Studies on chemical structure of complexes compounds formed by acid and alkaline components of pair medicines in decoction. Zhongguo Zhong Yao Za Zhi 34, 3054-3059 (2009).

11. Zheng, X. \& Sehgal, A. AKT and TOR signaling set the pace of the circadian pacemaker. Curr. Biol. 20, 1203-1208 (2010).

12. Luciano, A. K., Santana, J. M., Velazquez, H. \& Sessa, W. C. Akt1 controls the timing and amplitude of vascular circadian gene expression. J. Biol. Rhythms 32, 212-221 (2017).

13. Motohashi, H. et al. Polyporus and Bupleuri radix effectively alter peripheral circadian clock phase acutely in male mice. Nutr. Res. 43, 16-24 (2017).

14. Ramanthan, C. et al. Cell type-specific functions of Period genes revealed by novel adipocyte and hepatocyte circadian clock models. PLoS Genet 10, e1004244 (2014).

15. Ono, D. et al. Dissociation of Perl and Bmall circadian rhythms in the suprachiasmatic nucleus in parallel with behavioral outputs. Proc. Natl. Acad. Sci. U.S.A. 114, E3699-E3708 (2017).

16. Sato, M., Murakami, M., Node, K., Matsumura, R. \& Akashi, M. The role of the endocrine system in feeding-induced tissue-specific circadian entrainment. Cell Rep. 8, 393 (2014).

17. Luciano, A. K. et al. CLOCK phosphorylation by AKT regulates its nuclear accumulation and circadian gene expression in peripheral tissues. J. Biol. Chem. 293, 9126-9135 (2018).

18. Cao, Q. et al. Tenuifolin, a saponin derived from Radix Polygalae, exhibits sleep-enhancing effects in mice. Phytomedicine 23, 1797-1805 (2016).

19. Iida, M., Nakane Y., Yoshimura, T. \& Hirota, T. Effects of Cryptochrome-modulating compounds on circadian behavioral rhythms in zebrafish. J. Biochem. mvab096 (2021).

\title{
Acknowledgements
}

We thank Dr. Joseph S. Takahashi for providing Per2::Luciferase knockin mice.

\section{Author contributions}

T.Y., M.Z., and Y.N. designed the research. M.Z., K.K., H.A., Y.K., Y.N., J.C., R.N., T.N.O., N.K., A.S., and T.Y. conducted the experiments and analyzed the data. N.K., T.H., and T.M. provided new material and methods. M.Z., T.M., and T.Y. wrote the manuscript. All authors discussed the results and commented on the manuscript.

\section{Funding}

This work was supported in part by "Grant-in-Aid for Scientific Research (S)" (19H05643). WPI-ITbM is supported by the World Premier International Research Center Initiative (WPI), MEXT, Japan.

\section{Competing interests}

The authors declare no competing interests.

\section{Additional information}

Supplementary Information The online version contains supplementary material available at https://doi.org/ 10.1038/s41598-021-00499-w.

Correspondence and requests for materials should be addressed to T.Y.

Reprints and permissions information is available at www.nature.com/reprints.

Publisher's note Springer Nature remains neutral with regard to jurisdictional claims in published maps and institutional affiliations.

\begin{abstract}
Open Access This article is licensed under a Creative Commons Attribution 4.0 International License, which permits use, sharing, adaptation, distribution and reproduction in any medium or format, as long as you give appropriate credit to the original author(s) and the source, provide a link to the Creative Commons licence, and indicate if changes were made. The images or other third party material in this article are included in the article's Creative Commons licence, unless indicated otherwise in a credit line to the material. If material is not included in the article's Creative Commons licence and your intended use is not permitted by statutory regulation or exceeds the permitted use, you will need to obtain permission directly from the copyright holder. To view a copy of this licence, visit http://creativecommons.org/licenses/by/4.0/.
\end{abstract}

(C) The Author(s) 2021 